\title{
EXISTENCE RESULTS FOR A KIRCHHOFF-TYPE PROBLEM WITH SINGULARITY
}

\section{KHODABAKHSHI, S.M. VAEZPOUR, AND M. R. HEIDARI TAVANI}

Received 11 September, 2020

\begin{abstract}
In this work, using an infinitely many critical points theorem we establish the existence of a sequence of weak solutions for a Kirchhoff-type problem with singular term. This approach is based on variational methods and critical point theory.
\end{abstract}

2010 Mathematics Subject Classification: 35J35; 35J60

Keywords: singularity, Kirchhoff type problems, variational methods, critical point

\section{INTRODUCTION}

In 1883 , the stationary problem

$$
\rho \frac{\partial^{2} u}{\partial t^{2}}-\left(\frac{\rho_{0}}{h}+\frac{E}{2 L} \int_{0}^{L}\left|\frac{\partial u}{\partial x}\right|^{2} d x\right) \frac{\partial^{2} u}{\partial x^{2}}=0,
$$

was proposed by Kirchhoff [14] as an extension of the classical D'Alembert's wave equation for free vibrations of elastic strings. In recent years, the study of elliptic problems involving Kirchhoff type operators have been studied in many works, we refer to [1,3, 5-7, 16-18, 20, 22]. For instance, in [17], Molica Bisci and Pizzimenti considered the following problem

$$
\begin{cases}-\left(a+b \int_{\Omega}|\nabla u|^{p} d x\right) \Delta_{p} u+\alpha(x)|u|^{p-2} u=\lambda h(x) f(u) & \text { in } \Omega, \\ u=0 & \text { on } \partial \Omega .\end{cases}
$$

They obtained the existence of infinitely many weak solutions by using variational methods. Also, in [5], the authors studied the non-local problem

$$
\begin{cases}-M\left(\int_{\Omega}|\nabla u|^{p} d x\right) \Delta_{p} u=f(x, u) & \text { in } \Omega, \\ u=0 & \text { on } \partial \Omega .\end{cases}
$$

We gratefully thank the Iran National Science Foundation (INFS) for financial support. 
By using Browder Theorem, the writers proved the existence and uniqueness of solutions. On the other hand, singular elliptic problems have been intensively studied in the last decads. Among others, we mention the works [8-12, 15, 19,21]. Recently, motivated by this large interest, Ferrara and Molica Bisci in [8] studied the existence of at least one non-trivial weak solution for the following elliptic Dirichlet problem

$$
\left\{\begin{array}{l}
-\Delta_{p} u=\mu \frac{|u|^{p-2} u}{|x|^{p}}+\lambda f(x, u) \quad \text { in } \Omega, \\
\left.u\right|_{\partial \Omega}=0,
\end{array}\right.
$$

where $\lambda>0$ and $\mu \geq 0$ are two real parameters, $\Omega$ is a bounded domain in $\mathbb{R}^{N}(N \geq 2)$ containing the origin and with smooth boundary $\partial \Omega, 1<p<N$ and $f: \Omega \times \mathbb{R} \rightarrow \mathbb{R}$ is a Carathéodory function satisfying a suitable subcritical growth condition.

The aim of this paper is to investigate the existence of infinitely many weak solutions for the following problem

$$
\begin{cases}-M\left(\int_{\Omega}|\nabla u|^{p} d x\right) \Delta_{p} u+\frac{|u|^{q-2} u}{|x|^{q}}=\lambda f(x, u) & \text { in } \Omega, \\ u=0 & \text { on } \partial \Omega,\end{cases}
$$

where $\Delta_{p} u:=\operatorname{div}\left(|\nabla u|^{p-2} \nabla u\right)$ denotes the $p$-Laplace operator, $\Omega$ is a bounded domain in $\mathbb{R}^{N}(N \geq 2)$ containing the origin and with smooth boundary $\partial \Omega$, $1<q<N<p, M:[0,+\infty) \rightarrow R$ is a continuous function satisfying

$\left(\mathrm{f}_{1}\right)$ there are two positive constants $m_{0}, m_{1}$, such that

$$
m_{0} \leq M(t) \leq m_{1}, \quad \forall t \geq 0,
$$

and $f: \Omega \times \mathbb{R} \rightarrow \mathbb{R}$ is an $L^{1}$-Carathéodory function

Recall that a function $f: \Omega \times \mathbb{R} \rightarrow \mathbb{R}$ is said to be an $L^{1}$-Carathéodory function, if

$\left(\mathrm{C}_{1}\right)$ the function $x \mapsto f(x, t)$ is measurable for every $t \in \mathbb{R}$;

$\left(\mathrm{C}_{2}\right)$ the function $t \mapsto f(x, t)$ is continuous for a.e. $x \in \Omega$;

$\left(C_{3}\right)$ for every $\rho>0$ there exists a function $l_{\rho} \in L^{1}(\Omega)$ such that

$$
\sup _{|t| \leq \rho}|f(x, t)| \leq l_{\rho}(x),
$$

for a.e. $x \in \Omega$.

A special case of our main result is the following theorem.

Theorem 1. Assume that $f: \mathbb{R} \rightarrow \mathbb{R}$ is a non-negative continuous function such that

$$
\liminf _{\xi \rightarrow+\infty} \frac{f(\xi)}{\xi^{p-1}}=0 \quad \text { and } \quad \limsup _{\xi \rightarrow+\infty} \frac{\int_{0}^{\xi} f(t) d t}{\xi^{p}}=+\infty .
$$


Then, the problem

$$
\begin{cases}-\left(\frac{1+2\left(\int_{\Omega}|\nabla u|^{p} d x\right)^{2}}{1+\left(\int_{\Omega}|\nabla u|^{p} d x\right)^{2}}\right) \Delta_{p} u+\frac{|u|^{q-2} u}{|x|^{q}}=f(u) & \text { in } \Omega, \\ u=0 & \text { on } \partial \Omega\end{cases}
$$

admits a sequence of weak solutions which is unbounded in $X$.

\subsection{Preliminary considerations}

Let $\Omega$ be a bounded domain in $\mathbb{R}^{N}(N \geq 2)$ containing the origin and with smooth boundary $\partial \Omega$. Further, denote by $X$ the space $W_{0}^{1, p}(\Omega)$ endowed with the norm

$$
\|u\|:=\left(\int_{\Omega}|\nabla u(x)|^{p} d x\right)^{1 / p} .
$$

Also, let $\|\cdot\|_{1}$ denotes the usual norm of $L^{1}(\Omega)$; i.e.,

$$
\|u\|_{1}:=\int_{\Omega}|u(x)| d x .
$$

We recall classical Hardy's inequality, which says that

$$
\int_{\Omega} \frac{|u(x)|^{q}}{|x|^{q}} d x \leq \frac{1}{H} \int_{\Omega}|\nabla u(x)|^{q} d x, \quad(\forall u \in X),
$$

where $H:=\left(\frac{N-q}{q}\right)^{q}$; see, for instance, the paper [2].

Let us define $F(x, \xi):=\int_{0}^{\xi} f(x, t) d t$, for every $(x, \xi)$ in $\Omega \times \mathbb{R}$. Moreover we introduce the functional $I_{\lambda}: X \rightarrow \mathbb{R}$ associated with (1.1),

$$
I_{\lambda}(u):=\Phi(u)-\lambda \Psi(u),
$$

for every $u \in X$, where

$$
\Phi(u):=\frac{1}{p} \hat{M}\left(\|u\|^{p}\right)+\frac{1}{q} \int_{\Omega} \frac{|u(x)|^{q}}{|x|^{q}} d x,
$$

and

$$
\Psi(u):=\int_{\Omega} F(x, u(x)) d x,
$$

for every $u \in X$, where $\hat{M}(t):=\int_{0}^{t} M(s) d s, t \geq 0$. By standard arguments, one has that $\Phi$ is well defined (by Hardy's inequality), Gâteaux differentiable and sequentially weakly lower semicontinuous, and its Gâteaux derivative is the functional $\Phi^{\prime}(u) \in X^{*}$ given by

$$
\Phi^{\prime}(u)(v)=M\left(\int_{\Omega}|\nabla u|^{p} d x\right) \int_{\Omega}|\nabla u(x)|^{p-2} \nabla u(x) \nabla v(x) d x+\int_{\Omega} \frac{|u(x)|^{q-2}}{|x|^{q}} u(x) v(x) d x,
$$


for every $v \in X$ and clearly $\Phi$ is coercive. It is easy to prove that $\Phi$ is strongly continuous. On the other hand, standard arguments show that $\Psi$ is well defined and continuously Gâteaux differentiable functional whose Gâteaux derivative

$$
\Psi^{\prime}(u)(v)=\int_{\Omega} f(x, u(x)) v(x) d x,
$$

for every $v \in X$, is a compact operator from $X$ to the dual $X^{*}$.

Fixing the real parameter $\lambda$, a function $u: \Omega \rightarrow \mathbb{R}$ is said to be a weak solution of (1.1) if $u \in X$ and

$$
\begin{aligned}
& M\left(\int_{\Omega}|\nabla u|^{p} d x\right) \int_{\Omega}|\nabla u(x)|^{p-2} \nabla u(x) \nabla v(x) d x \\
& \quad+\int_{\Omega} \frac{|u(x)|^{q-2}}{|x|^{q}} u(x) v(x) d x-\lambda \int_{\Omega} f(x, u(x)) v(x) d x=0
\end{aligned}
$$

for every $v \in X$. Hence, the critical points of $I_{\lambda}$ are exactly the weak solutions of (1.1).

Our main tool to investigate the existence of infinitely many solutions for the problem (1.1) is a smooth version of [4, Theorem 2.1] which is a more precise version of Ricceri's variational principle [19, Theorem 2.5], which we now recall.

Theorem 2. Let $X$ be a reflexive real Banach space, let $\Phi, \Psi: X \rightarrow \mathbb{R}$ be two Gâateaux differentiable functionals such that $\Phi$ is sequentially weakly lower semicontinuous, strongly continuous and coercive, and $\Psi$ is sequentially weakly upper semicontinuous. For every $r>\inf _{X} \Phi$, put

$$
\begin{gathered}
\varphi(r):=\inf _{\Phi(u)<r} \frac{\left(\sup _{\Phi(v)<r} \Psi(v)\right)-\Psi(u)}{r-\Phi(u)}, \\
\gamma:=\liminf _{r \rightarrow+\infty} \varphi(r), \quad \text { and } \quad \delta:=\liminf _{r \rightarrow\left(\inf _{X} \Phi\right)^{+}} \varphi(r) .
\end{gathered}
$$

Then the following properties hold:

(a) For every $r>\inf _{X} \Phi$ and every $\left.\lambda \in\right] 0,1 / \varphi(r)[$, the restriction of the functional

$$
I_{\lambda}:=\Phi-\lambda \Psi
$$

to $\Phi^{-1}(]-\infty, r[)$ admits a global minimum, which is a critical point (local minimum) of $I_{\lambda}$ in $X$.

(b) If $\gamma<+\infty$, then for each $\lambda \in] 0,1 / \gamma[$, the following alternative holds: either

$\left(\mathrm{b}_{1}\right) I_{\lambda}$ possesses a global minimum, or

$\left(b_{2}\right)$ there is a sequence $\left\{u_{n}\right\}$ of critical points (local minima) of $I_{\lambda}$ such that

$$
\lim _{n \rightarrow+\infty} \Phi\left(u_{n}\right)=+\infty \text {. }
$$

(c) If $\delta<+\infty$, then for each $\lambda \in] 0,1 / \delta$, the following alternative holds: either 
$\left(c_{1}\right)$ there is a global minimum of $\Phi$ which is a local minimum of $I_{\lambda}$, or

$\left(c_{2}\right)$ there is a sequence $\left\{u_{n}\right\}$ of pairwise distinct critical points (local minima) of $I_{\lambda}$ which weakly converges to a global minimum of $\Phi$, with

$$
\lim _{n \rightarrow+\infty} \Phi\left(u_{n}\right)=\inf _{u \in X} \Phi(u) .
$$

\section{MAIN RESULTS}

Put

$$
k:=\sup _{u \in X, u \neq 0}\left(\frac{\max _{x \in \bar{\Omega}}|u(x)|}{\|u\|}\right) .
$$

Since the embedding $X \hookrightarrow C(\bar{\Omega})$ is compact, one has $k<+\infty$. Fix $x_{0} \in \Omega$ and $D>0$ such that $B\left(x_{0}, D\right) \subset \Omega$ and $\overline{B\left(x_{0}, D\right)}$ not containing the origin, where $B\left(x_{0}, D\right)$ denotes the ball with center at $x_{0}$ and radius $D$.

Put

$$
\begin{gathered}
\omega:=\frac{m_{1}}{p}\left[\left(\frac{2}{D}\right)^{p} m\left(D^{N}-\left(\frac{D}{2}\right)^{N}\right)\right], \\
\alpha:=\int_{B\left(x_{0}, \frac{D}{2}\right)} \frac{1}{|x|^{q}} d x, \quad \beta:=\left(\frac{2}{D}\right)^{q} \int_{B\left(x_{0}, D\right) \backslash B\left(x_{0}, \frac{D}{2}\right)} \frac{\left(D-\left|x-x_{0}\right|\right)^{q}}{|x|^{q}} d x,
\end{gathered}
$$

where $m:=\frac{\pi^{N / 2}}{\Gamma\left(1+\frac{N}{2}\right)}$. Here $\Gamma$ is the Gamma function defined by

$$
\Gamma(t):=\int_{0}^{+\infty} z^{t-1} e^{-z} d z \quad(\forall t>0)
$$

Put

$$
A:=\liminf _{\xi \rightarrow+\infty} \frac{\left\|l_{\xi}\right\|_{1}}{\xi^{p-1}}
$$

and

$$
B:=\limsup _{\xi \rightarrow+\infty} \frac{\int_{B\left(x_{0}, \frac{D}{2}\right)} F(x, \xi) d x}{\xi^{p}},
$$

where $l_{\xi} \in L^{1}(\Omega)$ satisfies condition $\left(\mathrm{C}_{3}\right)$ on $f(x, t)$ for every $\xi>0$.

Our main result is the following.

Theorem 3. Assume that $M:\left[0,+\infty\left[\rightarrow R\right.\right.$ is a continuous function satisfying $\left(\mathrm{f}_{1}\right)$. Also let $f: \Omega \times \mathbb{R} \rightarrow \mathbb{R}$ be an $L^{1}$-Carathéodory function such that

(i) $F(x, t) \geq 0$ for every $(x, t) \in \Omega \times \mathbb{R}^{+}$,

(ii) $A<\frac{1}{p \omega k^{p}} B$, where $k$ and $\omega$ are given by (2.1) and (2.2), respectively.

Then, for every $\lambda \in \Lambda:=] \frac{\omega}{B}, \frac{1}{p k^{p} A}[$, the problem (1.1) admits a sequence of weak solutions which is unbounded in $X$. 
Proof. Fix $\lambda \in] \frac{\omega}{B}, \frac{1}{p k^{p} A}[$. Our aim is to apply Theorem 2 part (b) with $X:=$ $W_{0}^{1, p}(\Omega)$ and where $\Phi$ and $\Psi$ are the functionals introduced in Section 2. As seen before, the functionals $\Phi$ and $\Psi$ satisfy the regularity assumptions requested in Theorem 2. Now, we look on the existence of critical points of the functional $I_{\lambda}:=\Phi-\lambda \Psi$ in $X$. To this end, we take $\left\{\xi_{n}\right\} \subset \mathbb{R}^{+}$such that $\lim _{n \rightarrow+\infty} \xi_{n}=+\infty$, and

$$
\lim _{n \rightarrow+\infty} \frac{\left\|l_{\xi_{n}}\right\|_{1}}{\xi_{n}^{p-1}}=A
$$

Set $r_{n}:=\frac{m_{0} \xi_{n}^{p}}{p k^{p}}$ for all $n \in \mathbb{N}$. From (2.1) we get

$$
\max _{x \in \bar{\Omega}}|u(x)| \leq k\|u\|,
$$

for every $u \in X$. Then, for each $u \in X$ with $\Phi(u)<r_{n}$, we have

$$
\max _{x \in \bar{\Omega}}|u(x)| \leq k\left(\frac{p}{m_{0}} \Phi(u)\right)^{1 / p}<k\left(\frac{p}{m_{0}} r_{n}\right)^{1 / p}=\xi_{n} .
$$

Then, since $\Phi(0)=\Psi(0)=0$, we have

$$
\begin{aligned}
\varphi\left(r_{n}\right) & =\inf _{\Phi(v)<r_{n}} \frac{\left(\sup _{\Phi(u)<r_{n}} \Psi(u)\right)-\Psi(v)}{r_{n}-\Phi(u)} \\
& \leq \frac{\sup _{\Phi(u)<r_{n}} \int_{\Omega} F(x, u(x)) d x}{r_{n}} \\
& \leq \frac{\xi_{n}\left\|l_{\xi_{n}}\right\|_{1}}{m_{0} \frac{\xi_{n}^{p}}{p k^{p}}} .
\end{aligned}
$$

Hence, it follows that

$$
\gamma \leq \liminf _{n \rightarrow+\infty} \varphi\left(r_{n}\right) \leq \frac{p}{m_{0}} k^{p} \liminf _{n \rightarrow+\infty} \frac{\left\|l_{\xi_{n}}\right\|_{1}}{\xi_{n}^{p-1}}=\frac{p}{m_{0}} k^{p} A<+\infty,
$$

since condition (ii) yields $A<+\infty$. Now, we claim that the functional $I_{\lambda}$ is unbounded from below. Let $\left\{d_{n}\right\}$ be a real sequence such that $\lim _{n \rightarrow+\infty} d_{n}=+\infty$ and

$$
\lim _{n \rightarrow+\infty} \frac{\int_{B\left(x_{0}, \frac{D}{2}\right)} F\left(x, d_{n}\right) d x}{d_{n}^{p}}=B .
$$

Further, for each $n \geq 1$, define $v_{n} \in X$ given by

$$
v_{n}(x):= \begin{cases}0, & x \in \Omega \backslash B\left(x_{0}, D\right), \\ d_{n}, & x \in B\left(x_{0}, \frac{D}{2}\right), \\ \frac{2 d_{n}}{D}\left(D-\left|x-x_{0}\right|\right), & x \in B\left(x_{0}, D\right) \backslash B\left(x_{0}, \frac{D}{2}\right) .\end{cases}
$$


By using condition (i), we infer

$$
\Psi\left(v_{n}\right)=\int_{\Omega} F\left(x, v_{n}(x)\right) d x \geq \int_{B\left(x_{0}, \frac{D}{2}\right)} F\left(x, d_{n}\right) d x,
$$

for every $n \geq 1$. Then, we have

$$
I_{\lambda}\left(v_{n}\right) \leq \omega d_{n}^{p}+\frac{\alpha+\beta}{q} d_{n}^{q}-\lambda \int_{B\left(x_{0}, \frac{D}{2}\right)} F\left(x, d_{n}\right) d x .
$$

If $B<+\infty$, let

$$
\delta \in] \frac{\omega}{\lambda B}, 1[.
$$

By (2.5), there exists $N_{\delta}$ such that

$$
\int_{B\left(x_{0}, \frac{D}{2}\right)} F\left(x, d_{n}\right) d x>\delta B d_{n}^{p}, \quad\left(\forall n>N_{\delta}\right) .
$$

Consequently, one has

$$
\begin{aligned}
I_{\lambda}\left(v_{n}\right) & <\omega d_{n}^{p}+\frac{\alpha+\beta}{q} d_{n}^{q}-\lambda \delta B d_{n}^{p} \\
& =(\omega-\lambda \delta B) d_{n}^{p}+\frac{\alpha+\beta}{q} d_{n}^{q},
\end{aligned}
$$

for every $n>N_{\delta}$. Then, it follows that

$$
\lim _{n \rightarrow+\infty} I_{\lambda}\left(v_{n}\right)=-\infty
$$

since $q<p$.

If $\mathrm{B}=+\infty$, let us consider $L>\frac{\omega}{\lambda}$. By (2.5), there exists $N_{L}$ such that

$$
\int_{B\left(x_{0}, \frac{D}{2}\right)} F\left(x, d_{n}\right) d x>L d_{n}^{p}, \quad\left(\forall n>N_{L}\right) .
$$

So, we have

$$
\begin{aligned}
I_{\lambda}\left(v_{n}\right) & <\omega d_{n}^{p}+\frac{\alpha+\beta}{q} d_{n}^{q}-\lambda L d_{n}^{p} \\
& =(\omega-\lambda L) d_{n}^{p}+\frac{\alpha+\beta}{q} d_{n}^{q} \quad\left(\forall n>N_{L}\right) .
\end{aligned}
$$

Taking into account the choice of $L$, also in this case, one has

$$
\lim _{n \rightarrow+\infty} I_{\lambda}\left(v_{n}\right)=-\infty,
$$

since $q<p$. Therefore owing to Theorem 2(b), the functional $I_{\lambda}$ admits an unbounded sequence $\left\{u_{n}\right\} \subset X$ of critical points. Then the problem (1.1) admits a sequence of weak solutions which is unbounded in $X$.

Among the consequences of Theorem 3, we point out the following result. 
Corollary 1. Let $f: \Omega \times \mathbb{R} \rightarrow \mathbb{R}$ be an $L^{1}$-Carathéodory function. Assume that condition (i) of Theorem 3 holds. Further, require that

(iii) $A<\frac{1}{p k^{p}}$ and $B>\omega$, where $k$ and $\omega$ are given by (2.1) and (2.2), respectively. Then the following problem

$$
\begin{cases}-M\left(\int_{\Omega}|\nabla u|^{p} d x\right) \Delta_{p} u+\frac{|u|^{q-2} u}{|x|^{q}}=f(x, u), & \text { in } \Omega, \\ u=0, & \text { on } \partial \Omega,\end{cases}
$$

admits a sequences of weak solutions which is unbounded in $X$.

Remark 1. We note that assumption (ii) in Theorem 3 could be replaced by the following more general hypothesis:

(ii') There exists two positive sequences $\left\{a_{n}\right\}$ and $\left\{b_{n}\right\}$ such that

$$
\frac{1}{p} \int_{0}^{\frac{\omega}{m_{1}} a_{n}{ }^{p}} M(s) d s+\frac{(\alpha+\beta)}{q} a_{n}^{q}<\frac{m_{0} b_{n}^{p}}{p k^{p}}, \quad(\forall n \geq 1)
$$

and $\lim _{n \rightarrow+\infty} b_{n}=+\infty$ such that

$$
\widetilde{A}<\frac{B}{\omega},
$$

where $k, \omega$ and $\alpha, \beta$ are given by (2.1), (2.2) and (2.3), respectively, and

$$
\widetilde{A}:=\lim _{n \rightarrow+\infty} \frac{b_{n}\left\|l_{b_{n}}\right\|_{1}-\int_{B\left(x_{0}, \frac{D}{2}\right)} F\left(x, a_{n}\right) d x}{\frac{m_{0} b_{n}^{p}}{p k^{p}}-\frac{1}{p} \int_{0}^{\frac{\omega}{m_{1}} a_{n}^{p}} M(s) d s-\frac{(\alpha+\beta)}{q} a_{n}^{q}} .
$$

Then, for every

$$
\lambda \in] \frac{\omega}{B}, \frac{1}{\widetilde{A}}[
$$

the problem (1.1) admits a sequence of weak solutions which is unbounded in $X$.

Indeed, from (ii') we obtain (ii), by choosing $a_{n}=0$ for all $n \in \mathbb{N}$. Moreover, if we assume (ii') instead of (ii) and set $r_{n}:=\frac{m_{0} b_{n}^{p}}{p k^{p}}$ for every $n \geq 1$, one has

$$
\begin{aligned}
\varphi\left(r_{n}\right) & :=\inf _{\Phi(v)<r_{n}} \frac{\left(\sup _{\Phi(u)<r_{n}} \int_{\Omega} F(x, u(x)) d x\right)-\int_{\Omega} F(x, v(x)) d x}{r_{n}-\Phi(v)} \\
& \leq \frac{b_{n}\left\|l_{b_{n}}\right\|_{1}-\int_{\Omega} F\left(x, v_{n}(x)\right) d x}{\frac{m_{0} b_{n}^{p}}{p k^{p}}-\Phi\left(v_{n}\right)}
\end{aligned}
$$




$$
\leq \frac{b_{n}\left\|l_{b_{n}}\right\|_{1}-\int_{B\left(x_{0}, \frac{D}{2}\right)} F\left(x, a_{n}\right) d x}{\frac{m_{0} b_{n}^{p}}{p k^{p}}-\frac{1}{p} \int_{0}^{\frac{\omega}{m_{1}} a_{n}{ }^{p}} M(s) d s-\frac{(\alpha+\beta)}{q} a_{n}^{q}},
$$

by choosing

$$
v_{n}(x):= \begin{cases}0, & x \in \Omega \backslash B\left(x_{0}, D\right), \\ a_{n}, & x \in B\left(x_{0}, \frac{D}{2}\right), \\ \frac{2 a_{n}}{D}\left(D-\left|x-x_{0}\right|\right), & x \in B\left(x_{0}, D\right) \backslash B\left(x_{0}, \frac{D}{2}\right),\end{cases}
$$

for every $n \geq 1$. Therefore, since by assumption (ii') one has $\widetilde{A}<+\infty$, we obtain

$$
\gamma \leq \liminf _{n \rightarrow+\infty} \varphi\left(r_{n}\right) \leq \widetilde{A}<+\infty .
$$

From now on, arguing as in the proof of Theorem 3, the conclusion follows.

Now, we present the other main result. First, put

$$
A^{\prime}:=\liminf _{\xi \rightarrow 0^{+}} \frac{\left\|l_{\xi}\right\|_{1}}{\xi^{p-1}}, \quad \quad B^{\prime}:=\limsup _{\xi \rightarrow 0^{+}} \frac{\int_{B\left(x_{0}, \frac{D}{2}\right)} F(x, \xi) d x}{\xi^{p}} .
$$

Arguing as in the proof of Theorem 3 but using conclusion (c) of Theorem 2 instead of (b), the following result holds.

Theorem 4. Assume that $f: \Omega \times \mathbb{R} \rightarrow \mathbb{R}$ be an $L^{1}$-Carathéodory function such that hypothesis (i) in Theorem 3 holds, and

(iv) $A^{\prime}<\frac{m_{0}}{p \omega k^{p}} B^{\prime}$.

Then, for every $\left.\lambda \in \Lambda^{\prime}:=\right] \frac{\omega}{B^{\prime}}, \frac{m_{0}}{p^{p} A^{\prime}}[$, the problem (1.1) has a sequence of weak solutions, which strongly converges to zero in $X$.

Proof. Fix $\lambda \in \Lambda^{\prime}$. We take $\Phi, \Psi$ and $I_{\lambda}$ as in Section 2. Now, as it has been pointed out before, the functionals $\Phi$ and $\Psi$ satisfy the regularity assumptions reqired in Theorem 2. As first step, we will prove that $\lambda<1 / \delta$. Then, let $\left\{\xi_{n}\right\}$ be a sequence of positive numbers such that $\lim _{n \rightarrow+\infty} \xi_{n}=0$ and

$$
\lim _{n \rightarrow+\infty} \frac{\left\|l_{\xi_{n}}\right\|_{1}}{\xi_{n}^{p}}=A^{\prime}
$$

By the fact that $\inf _{X} \Phi=0$ and the definition of $\delta$, we have $\delta:=\liminf _{r \rightarrow 0^{+}} \varphi(r)$. Put $r_{n}:=\frac{m_{0} \xi_{n}^{p}}{p k^{p}}$ for all $n \in \mathbb{N}$. Then, for all $u \in X$ with $\Phi(u)<r_{n}$, taking (2.4) into account, one has $\|u\|_{\infty}<\xi_{n}$. Thus, for all $n \in \mathbb{N}$,

$$
\varphi\left(r_{n}\right) \leq \frac{\sup _{\Phi(u)<r_{n}} \Psi(u)}{r_{n}} \leq \frac{p k^{p}}{m_{0}} \frac{\left\|l_{\xi_{n}}\right\|_{1}}{\xi_{n}^{p-1}} .
$$


Hence,

$$
\delta \leq \liminf _{n \rightarrow+\infty} \varphi\left(r_{n}\right) \leq \frac{p k^{p}}{m_{0}} \liminf _{n \rightarrow+\infty} \frac{\left\|l_{\xi_{n}}\right\|_{1}}{\xi_{n}^{p-1}}=\frac{p k^{p} A^{\prime}}{m_{0}}<+\infty,
$$

and therefore $\left.\Lambda^{\prime} \subset\right] 0,1 / \delta[$.

Let $\lambda$ be fixed. We claim that the functional $I_{\lambda}$ does not have a local minimum at zero. Let $\left\{d_{n}\right\}$ be a sequence of positive numbers such that $\lim _{n \rightarrow+\infty} d_{n}=0$ and

$$
\lim _{n \rightarrow+\infty} \frac{\int_{B\left(x_{0}, \frac{D}{2}\right)} F\left(x, d_{n}\right) d x}{d_{n}^{p}}=B^{\prime} .
$$

For all $n \in \mathbb{N}$, let $v_{n} \in X$ defined by (2.6) with the above $d_{n}$. Now, with the same argument as in the proof of Theorem 3, we achieve $I_{\lambda}\left(v_{n}\right)<0$ for every $n \in \mathbb{N}$ large enough. Then, since $\lim _{n \rightarrow+\infty} I_{\lambda}\left(v_{n}\right)=I_{\lambda}(0)=0$, we see that zero is not a local minimum of $I_{\lambda}$. This, together with the fact that zero is the only global minimum of $\Phi$, we deduce that the energy functional $I_{\lambda}$ does not have a local minimum at the unique global minimum of $\Phi$. Therefore, by Theorem 2(c), there exists a sequence $\left\{u_{n}\right\}$ of critical points of $I_{\lambda}$ which converges weakly to zero. In view of the fact that the embedding $X \hookrightarrow C(\bar{\Omega})$ is compact, we know that the critical points converge strongly to zero, and the proof is complete.

We end this paper with the following example to illustrate our results.

Example 1. Let $r>0$ be a real number and $\left\{t_{n}\right\},\left\{s_{n}\right\}$ be two strictly increasing sequences of real numbers that defined by induction

$$
t_{1}=r, s_{1}=2 r
$$

and for $n \geq 1$,

$$
\begin{gathered}
t_{2 n}=\left(2^{2 n+1}-1\right) t_{2 n-1}, \quad t_{2 n+1}=\left(2-\frac{1}{2^{2 n+1}}\right) t_{2 n}, \\
s_{2 n}=\frac{t_{2 n}}{2^{n}}=\left(2-\frac{1}{2^{2 n}}\right) s_{2 n-1}, \quad s_{2 n+1}=2^{n+1} t_{2 n+1}=\left(2^{2 n+2}-1\right) s_{2 n} .
\end{gathered}
$$

Let $f: \Omega \times \mathbb{R} \rightarrow \mathbb{R}$ be the function defined by

$f(x, t):= \begin{cases}2 \varphi(x) t, & (x, t) \in \Omega \times\left[0, t_{1}\right], \\ \varphi(x)\left(s_{n-1}+\frac{s_{n}-s_{n-1}}{t_{n}-t_{n-1}}\left(t-t_{n-1}\right)\right), & (x, t) \in \Omega \times\left[t_{n-1}, t_{n}\right] \text { for some } n>1,\end{cases}$

where $\varphi: \Omega \rightarrow \mathbb{R}$ is a positive continuous function with $0<m \leq \varphi(x) \leq M$. Then $f$ is an $L^{1}$-Carathéodory function and since $f(x, t)$ is strictly increasing with respect to $t$ argument at every $x \in \Omega$, the function $l_{\xi}(x):=f(x, \xi)$ satisfies in condition $\left(\mathrm{C}_{3}\right)$ on $f$; i.e.,

$$
\sup _{|t| \leq \xi}|f(x, t)| \leq l_{\xi}(x), \quad \text { for a.e. } x \in \Omega .
$$


Arguing as in [13], we have

$$
\limsup _{\xi \rightarrow+\infty} \frac{\int_{B\left(x_{0}, \frac{D}{2}\right)} F(x, \xi) d x}{\xi^{\frac{7}{3}}}=+\infty, \quad \liminf _{\xi \rightarrow+\infty} \frac{\left\|l_{\xi}\right\|_{1}}{\xi^{\frac{4}{3}}}=0,
$$

for every $x_{0} \in \Omega$ and $D>0$ such that $B\left(x_{0}, D\right) \subset \Omega$ and $\overline{B\left(x_{0}, D\right)}$ not containing the origin, where $\Omega$ is a bounded domain in $\mathbb{R}^{2}$ containing the origin and with smooth boundary $\partial \Omega$. Hence, by Theorem 3 , for every $\lambda \in] 0,+\infty[$, the following problem

$$
\begin{cases}-M\left(\int_{\Omega}|\nabla u|^{\frac{7}{3}} d x\right) \Delta_{\frac{7}{3}} u+\frac{|u|^{\frac{-1}{2}} u}{|x|^{\frac{3}{2}}}=\lambda f(x, u), & \text { in } \Omega, \\ u=0, & \text { on } \partial \Omega,\end{cases}
$$

possesses an unbounded sequence of weak solutions in $W_{0}^{1, \frac{7}{3}}(\Omega)$.

\section{ACKNOWLEDGEMENT}

We would like to show our gratitude to the anonymous reviewer for their valuable comments and suggestions to improve the paper.

\section{REFERENCES}

[1] C. O. Alves, F. J. S. A. Correa, and T. M. Ma, "Positive Solution for a quasi-linear elliptic equation of Kirchhoff type." Comput Math Appl., vol. 49, no. 1, pp. 85-93, 2005, doi: 10.1016/j.camwa.2005.01.008.

[2] J. P. G. Azorero and I. P. Alonso, "Hardy inequalities and some critical elliptic and parabolic problems," J Differ Equ., vol. 144, no. 2, pp. 441-476, 1998, doi: 10.1006/jdeq.1997.3375.

[3] A. Bensedik and M. Bouchekif, "On an elliptic equation of Kirchhoff type with a Potential asympt otically linear at infinity." Math. Comput. Modelling., vol. 49, no. 5-6, pp. 1089-1096, 2009, doi: 10.1016/j.mcm.2008.07.032.

[4] G. Bonanno and G. Molica Bisci, "Infinitely many solutions for a boundary value problem with discontinuous nonlinearities," Bound. Value Probl., vol. 2009, pp. 1-20, 2009, doi: $10.1155 / 2009 / 670675$.

[5] T. Bouali and R. Guefaifia, "Existence and uniqueness of weak solution for a non-local problem involving the p-Laplacian." Int. J.Pure Appl. Math., vol. 98, no. 1, pp. 11-21, 2015, doi: 10.12732/ijpam.v98i1.2.

[6] N. T. Chung, "Multiple Solutions for a $p(x)$-Kirchhoff type equation with Sing-Charging nonlinearities," Complex Var. Elliptic Equ.

[7] N. T. Chung, "Multiplicity results for a class of $p(x)$-Kirchhoff type equations with combined nonlinearities," Electron. J. Qual. Theory Differ. Equ., vol. 2012, no. 42, pp. 1-13, 2012, doi: 10.14232/ejqtde.2012.1.42.

[8] M. Ferrara and G. M. Bisci, "Existence results for elliptic problems with Hardy potential," Bull. Sci. Math., vol. 138, no. 7, pp. 846-859, 2014, doi: 10.1016/j.bulsci.2014.02.002.

[9] M. Ghergu and V. D. Rădulescu, "Sublinear singular elliptic problems with two parameters," $J$ Differ Equ., vol. 195, no. 2, pp. 520-536, 2003, doi: 10.1016/S0022-0396(03)00105-0. 
[10] M. Ghergu and V. D. Rădulescu, "Multiparameter bifurcation and asymptotics for the singular Lane-Emden-Fowler equation with a convection term," Proc. Roy.Soc. Edinburgh Sect. A., vol. 135, no. 1, pp. 61-83, 2005, doi: 10.1017/S0308210500003760.

[11] M. Ghergu and V. D. Rădulescu, "Ground state solutions for the singular Lane-Emden-Fowler equation with sublinear convection term," J. Math. Anal. Appl., vol. 333, no. 1, pp. 265-273, 2007, doi: 10.1016/j.jmaa.2006.09.074.

[12] M. Ghergu and V. D. Rădulescu, Singular Elliptic Problems: Bifurcation and Asymptotic Analysis. Oxford: Oxford: Oxford University Press, 2008.

[13] S. Khalkhali, S. Heidarkhani, and AbdolrahmanRazani, "Infinitely many weak solutions for a forth-order boundary-value problem," Electron. J. Diff. Equ., vol. 2012, no. 164, pp. 1-14, 2012.

[14] G. Kirchhoff, Vorlesungen über mathematische Physik. Mechanik. $2^{\text {te }}$ Aufl. Leipzig. Teubner (1877)., 1877.

[15] A. Kristály and C. Varga, "Multiple solutions for elliptic problems with singular and sublinear potentials," Proc. Am. Math. Soc., vol. 135, no. 7, pp. 2121-2126, 2007, doi: 10.1090/S00029939-07-08715-1.

[16] T. Ma, "Remarks on an elliptic equation of Kirchhoff type," Nonlinear Anal Theory Methods Appl, vol. 63, no. 5-7, pp. 1967-1977, 2005, doi: 10.1016/j.na.2005.03.021.

[17] G. Molica Bisci and P. F. Pizzimenti, "Sequences of weak solutions for non-local elliptic problems with Dirichlet boundary condition.” Proc. Edinb. Math. Soc., II. Ser., vol. 57, no. 3, pp. 779-809, 2014, doi: 10.1017/S0013091513000722.

[18] K. Perera and Z. Zhang, "Nontrivial solutions of Kirchhoff-type problems via the Yang index," $J$. Differ. Equations, vol. 221, no. 1, pp. 246-255, 2006, doi: 10.1016/j.jde.2005.03.006.

[19] B. Ricceri, "A general variational principle and some of its applications," J. Comput. Appl. Math., vol. 113, no. 1-2, pp. 401-410, 2000, doi: 10.1016/S0377-0427(99)00269-1.

[20] B. Ricceri, "On an elliptic Kirchhoff-type problem depending on two parameters," J. Glob. Optim., vol. 46, no. 4, pp. 543-549, 2010, doi: 10.1007/s10898-009-9438-7.

[21] V. D. Rădulescu, "Combined effects in nonlinear singular elliptic problems with convenction," Rev. Roumaine Math. Pures Appl, vol. 53, no. 5, pp. 543-553, 2008.

[22] J. J. Sun and C. L. Tang, "Existence and multiplicity of solutions for Kirchhoff type equations," Nonlinear Anal Theory Methods Appl, vol. 74, no. 4, pp. 1212-1222, 2011, doi: 10.1016/j.na.2010.09.061.

\section{Authors' addresses}

\section{Khodabakhshi}

Department of Mathematics and Computer Science, Amirkabir University of Technology, Tehran, Iran

E-mail address: m.khodabakhshi11@gmail.com

S.M. Vaezpour

Department of Mathematics and Computer Science, Amirkabir University of Thechnology, Tehran, Iran

E-mail address: vaez@aut.ac.ir

\section{R. Heidari Tavani}

Department of Mathematics, Ramhormoz Branch, Islamic Azad University, Ramhormoz, Iran

E-mail address: m.reza.h56@gmail.com 